\title{
All-Optical Clock Division Using Period-one Oscillation of Optically Injected Semiconductor Laser
}

\author{
Shengxiao Niu \\ Science and technology research and development center \\ of Handan polytechnic college \\ Handan, China \\ nsx618@sina.com \\ Mingjiang Zhang \\ Key Laboratory of Advanced Transducers and Intelligent \\ Control Systems(Taiyuan University of Technology), \\ Ministry of Education, \\ Taiyuan Shanxi \\ zhangmingjing@gmail.com
}

\begin{abstract}
Nonlinear dynamics of a semiconductor laser subjected external optical is applied to all-optical clock division. By properly adjusting the injection conditions, the divide-by-two clock division and the divide-by-three clock division are achieved base on the period-one oscillation. The divide-by-two clock division and divide-by-three clock division can be implemented within different input signal frequency ranges: from $11.0 \mathrm{GHz}$ to $20.0 \mathrm{GHz}$ and from 16.5 GHz to $20.0 \mathrm{GHz}$, respectively, by adjusting the average power of the injection optical pulses. In addition, our experiment shows that the phase noise of the divided clock signals is below $-100 \mathrm{dBc} / \mathrm{Hz}$. The division process can be controlled by the power and the wavelength detuning of the injected optical pulses. A large locking range of $1.0 \mathrm{GHz}$ is measured in second clock division, and $1.8 \mathrm{GHz}$ is measured in third clock division.
\end{abstract}

Keywords-semiconductor lasers; nonlinear optics; clock division; optical injection; time-division multiplexing.

\section{INTRODUCTION}

Optical networks employing optical time-division-multiplexing (OTDM) will be required to accommodate increasing traffic in communications. A reliable and convenient approach to extracting individual channel signal has an important role in the OTDM system (so called clock division). Various technique base on different devices for all-optical clock division have been demonstrated, including semiconductor optical amplify $(\mathrm{SOA})^{[1-4]}$, fiber laser diode ${ }^{[5]}$, semiconductor laser ${ }^{[6-8]}$ and so on. However, the semiconductor laser is the most potential device used for all optical clock division, since the setup of all optical clock division bases on semiconductor laser is simple and reliable. A number of techniques have been demonstrated for clock division using semiconductor laser. They include the use of mode-locked lasers ${ }^{[6-8]}$, optoelectronic feedback loop techniques [9], and optoelectronic oscillation ${ }^{[10]}$. All-optical clock division from $10 \mathrm{GHz}$ to $5 \mathrm{GHz}$ and

\author{
Shuhui Shi \\ The physics department of \\ Handan collage \\ Handan, China \\ Shish987@163.com \\ Chao Chen \\ The software department of \\ Handan, China \\ Chengchao519@126.com
}

19.6 GHz to $9.8 \mathrm{GHz}$ using the techniques of mode-locked lasers has been demonstrated, and the clock division is dependent on the relaxation oscillation of the semiconductor laser diode ${ }^{[6-7]}$. All-optical clock division from $12.4 \mathrm{GHz}$ to $6.2 \mathrm{GHz}$ achieved under the resonance frequency of the laser ${ }^{[8]}$. Microwave clock division from $18.56 \mathrm{GHz}$ to $9.28 \mathrm{GHz}$ has been demonstrated, and the clock division is dependent on the period-two state of external optical injected semiconductor laser ${ }^{[11]}$. The reporter expected to achieve all-optical clock division using this method. All above papers only reported a signal frequency or a small frequency range clock division.

In this letter, we reported a new technique for clock division by the nonlinear dynamics of external optical injected semiconductor laser. Utilizing the period-one oscillation, we achieved second clock division in the frequency range from $11.0 \mathrm{GHz}$ to $20.0 \mathrm{GHz}$, and achieved third clock division in the frequency range from $16.5 \mathrm{GHz}$ to $20.0 \mathrm{GHz}$.

\section{EXPERIMENTS}

The experimental configuration on all-optical clock division is shown in Figure1. The master laser is a $1.55 \mathrm{um}$ distributed feedback laser diode (DFB-LD), and it is biased at $24 \mathrm{~mA}$ which is 1.4 times the threshold current. The slave laser is a 1.55 um Fabry-perot laser diode (FP-LD), and it is biased at $21 \mathrm{~mA}$ which is 1.5 times the threshold current. A radio-frequency synthesizer (Agilent E8257D) through an electro-absorption modulator (EAM) modulated the continue-wave light emitted by the DFB-LD to pulses. After amplified by an erbium-doped fiber amplifier (EDFA), the output optical pulses inject into the FP-LD through an optical circulator. The EDFA is employed to adjust the injected optical power level, and the polarization controller (PC) is used to control the coupling efficiency of the injected light. The output pulses from the FP-LD amplified by another EDFA, and then measured with a sampling oscilloscope (Agilent 86100B) 
and a spectrum analyzer (Agilent E4407B). An optical spectrum analyzer (Agilent 86140B) is employed to observe the optical spectrum of the output pulses.

\section{RESULTS}

Without optical injection, the FP-LD is operating CW conditions with multiple modes, and its central wavelength is $1550.26 \mathrm{~nm}$, shown as Figure 2(a). Injection locking occurs at this laser cavity mode when the wavelength of the injected external optical is set at $1550.23 \mathrm{~nm}$, and then the FP-LD is locked by the injected optical signal. Meanwhile, the central mode will be strengthened; other modes will be suppressed, shown as Figure 2(b)

The Wavelength detuning, defined as the DFB-LD's wavelength offset from the FP-LD's free-running wavelength, is fixed at $0.03 \mathrm{~nm}$. When the power of the injected optical is fixed at $-3.97 \mathrm{dBm}$, appeared period-one oscillating, and the fundamental frequency of the period-one oscillating is $6.0 \mathrm{GHz}$, shown as Figure 3. The period-one oscillation is dependent on the beating of the two optical frequencies ${ }^{[11]}$. The fundamental frequency of the period-one oscillating changed with the power of the external optical and the wavelength detuning of the external and the optical of FP-LD. Shown as Figure 4.

The linewidths of the period-one oscillation generated by the nonlinear dynamics are rather broad due to noise. Applying the pulses with repetition rate of $12 \mathrm{GHz}$ modulation the $\mathrm{CW}$ optical of the injected, the linewidths can be substantially narrowed; the subharmonica frequency and the fundamental frequency will be locked, achieved second frequency clock division. The repetition rate of the injected optical pulses is set at $12 \mathrm{GHz}$, and the repetition rate of the output pulse is $6 \mathrm{GHz}$, as shown in Figure 5. Figure 5(a) shows the frequency domain spectrum of the injected optical pulses. Figure 5(b) shows the frequency domain spectrum of the output optical pulses. The intensity at $6 \mathrm{GHz}$ is $-20 \mathrm{~dB}$ greater than that at $12 \mathrm{GHz}$. The dependence of the phase noise of the output clock signal on the input optical signal parameter is also investigated. Figure 6 shows the measured phase noise of the output clock signal as a function of the input clock frequency when the average power of the injected pulse signal and the wavelength detuning were fixed at $0.03 \mathrm{~nm}$. The results indicated that a low phase noise of output clock signal can be obtained even when the repetition rate of the input clock signal is changed. If we define the detuning range as the phase noise of output clock lower than $-100 \mathrm{dBc} / \mathrm{Hz}$, the detune range is as high as $1 \mathrm{GHz}$.

When the injected optical is modulated at the repetition rate of $18.0 \mathrm{GHz}$, the linewidths can be substantially narrowed, achieved third frequency clock division. The repetition rate of the injected optical pulses is set at $12 \mathrm{GHz}$, and the repetition rate of the output pulse is $6 \mathrm{GHz}$, as shown in Figure 7. Figure 7(a) shows the frequency domain spectrum of injected pulse. Figure 7(b) shows the frequency domain spectrum of output pulse.

\section{DISCUSSIONS}

The fundamental frequency of the period-one oscillation increased versus the increase of the power of the injected optical $[10,11]$. The injected optical is modulated by the pulse with the frequency approached the second harmonic of the period-one oscillation, the sub harmonica frequency and the fundamental frequency will be locked, achieved second frequency clock division. The second frequency clock division is achieved in the frequency offset range about $1 \mathrm{GHz}$ detuned about the second harmonic frequency of the period-one with the repetition rate, show as Figure 9. The injected optical is modulated by the pulse with the frequency approached the third harmonic of the period-one oscillation, the sub harmonica frequency and the fundamental frequency will be locked, achieved third frequency clock division. When the third harmonic of the period-one oscillation is approach the repetition rate, the subharmonica frequency and the fundamental frequency will be locked, third frequency clock division achieved. The third frequency clock division is achieved in the frequency range about $1.8 \mathrm{GHz}$ detuned about the third harmonic frequency of the period-one with the repetition rate. On the condition of our lab, achieve second frequency clock division in the frequency range from $11 \mathrm{GHz}$ to $20 \mathrm{GHz}$, and achieve third frequency clock division in the frequency range from $16.5 \mathrm{GHz}$ to $20 \mathrm{GHz}$. This technique can achieve second frequency clock division and third frequency clock division through change the injected power of the pulse and the wavelength detuning of the two optical.

The setup is expected to be capable of achieve second frequency clock division and third frequency clock division in the more ultra fast repetition rate. When the fourth or fifth harmonic frequency of the period-one oscillating approached the rate repetition of signal pulse, we may obtain the quarter or quintile frequency clock division of the signal pulse.

\section{CONCLUSIONS}

All-optical clock frequency division from $12.0 \mathrm{GHz}$ to $5.0 \mathrm{GHz}$ has been demonstrated with an FP-LD using the external optical pulses injected. The division process can be controlled by the average power of the pulses an the wavelength detuning. In the different average power of injected and the wavelength detuning achieve second frequency clock division in the frequency range from $11 \mathrm{GHz}$ to $20 \mathrm{GHz}$, and achieve third frequency clock division in the frequency range from $16.5 \mathrm{GHz}$ to $20 \mathrm{GHz}$. When external optical injected into semiconductor laser, will obtain period-one oscillation, And the fundamental frequency changed with the power of the external optical and the wavelength detuning. Using a same average power pulse take place the external optical injected into semiconductor laser diode, when the pulses repetition rate approach the second harmonica achieved second frequency clock division, and when the repetition rage approach the third harmonica achieve third frequency clock division. 


\section{ACKNOWLEDGMENT}

This work was Supported by the Key Laboratory of Advanced Transducers and Intelligent Control System (Taiyuan University of Technology), Ministry of Education, Taiyuan, China(No: 201301)

\section{REFERENCES}

[1] A. E. Kelly, R. J. Manning, A. J. Poustie, etal, "All-optical clock division at 10 and $20 \mathrm{GHz}$ in a semiconductor optical amplifier based nonlinear loop mirror," Electron. Lett., 1998, 34(13): 1337-1339.

[2] L. Xu, M. Y. Yao, B. C. Wang, etal, "All-optical clock division with mode-locked figure-eight laser based on the slow carrier recovery rate in semiconductor optical amplifier," IEEE Photon. Techn. Lett., 2002, 14 (3): 402-404.

[3] K. K. Chow, C. Shu, Y. M. Yang, H. F. Liu, "Optical control of period doubling in a gain-switched Fabry-Perot laser diode and its application in all-optical clock division," IEE Proc-Optoelectron., 2003, 150 (3): 239-245.

[4] K. K. Chow, C. Chu, and H. F. Liu, "All-optical control of clock frequency division using injection-locked Fabry-Perot laser diode,” Electron. Lett., 2003, 39 (15): 1136-1138.
[5] Y. M. Yang, H. F. Liu, Y. Mastsui, "Scheme for all-optical clock division based on period doubling in semiconductor lasers,' Electron. Lett., 2000, 36(22): 1852-1854.

[6] A. B. Wang, Y. C. Wang, H. C. He, "Enhancing the bandwidth of the optical chaotic signal generated by a semiconductor laser with optical feedback" IEEE Photon. Techn. Lett., 2008, 20(19): 1633-1635.

[7] T. B. Simpson, J. M. Liu, K. F. Huang, etal, "Nonlinear dynamics induced by external optical injection in semiconductor lasers," Quantum Semiclass. 1997, 9: 765-784.

[8] S. K. Hwang, J. M. Liu, "Dynamical characteristics of optically injected semiconductor laser," Opto. Commun. 2000, 183: 195-205.

[9] S. K. Hwang, J. M. Liu, J. K. White, "Characteristics of Period-One Oscillations in Semiconductor Lasers Subject to Optical Injection,” IEEE J. Sel. Topics Quantum Electron., 2004, 10(5): 974-981

[10] Z. C. Chun, J. M. Liu, "Frequency modulation on single sideband using controlled dynamics of optically injected semiconductor laser," IEEE J. Quantum Electron., 2006, 42(7): 699-704.

[11] S. X. Niu, M. J. Zhang, Y. An, etal, "Frequency-tunable all-optical clock division using semiconductor laser subjected to external optical injection," Acta Phys. Sin., 2008, 57(11): 6998-7004.

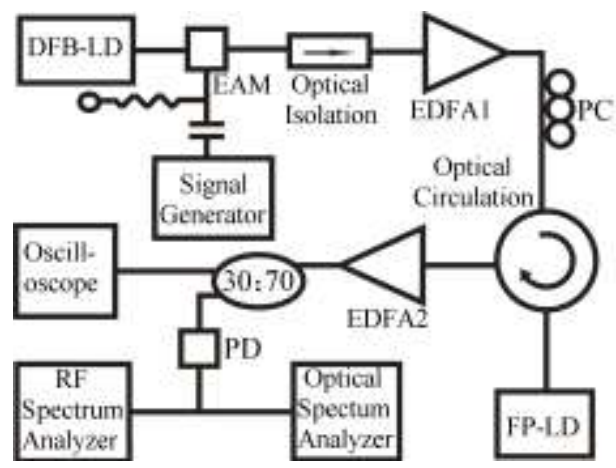

Figure 1. Experimental setup
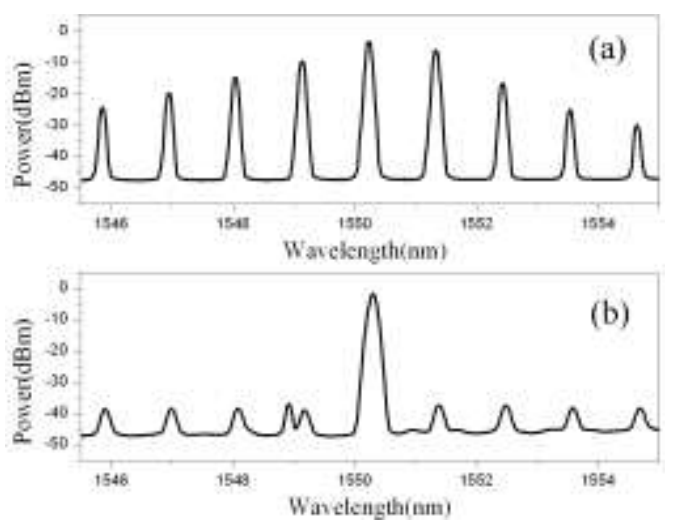

Figure 2. (a) Spectrum of FP-LD without optical injected (b) Spectrum of FP-LD with optical injected 


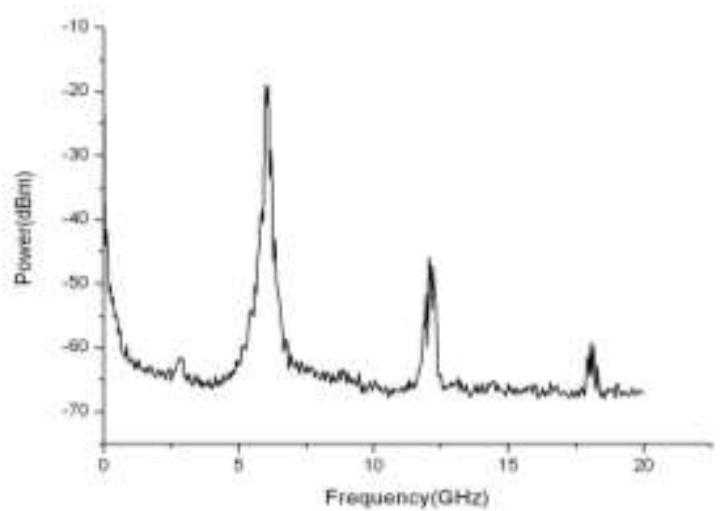

Figure 3. Frequency spectrum of period-one oscillation

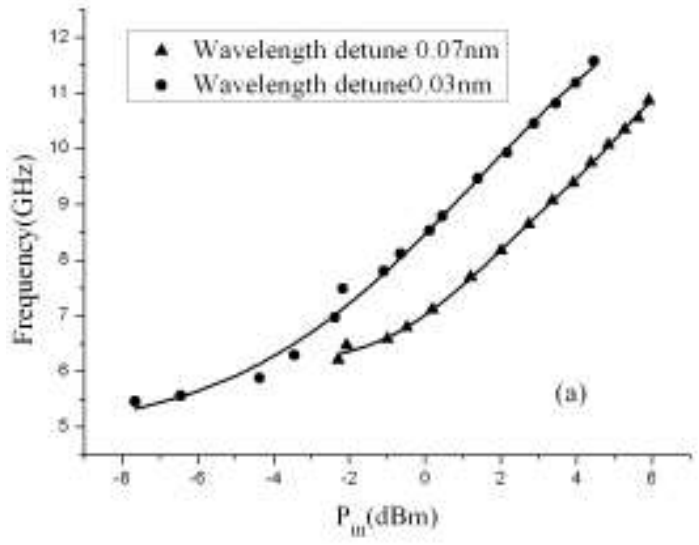

Figure 4. Fundamental frequency of period-one oscillation versus the injected optical power
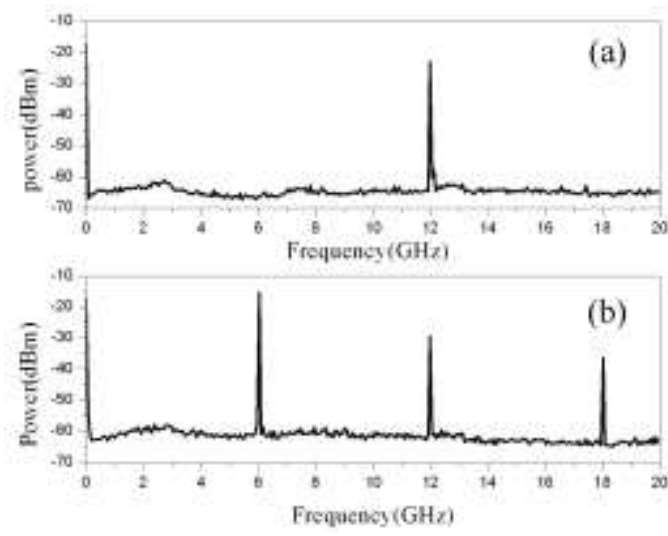

Figure 5. (a) Frequency spectrum of input $12 \mathrm{GHz}$ pulses (b) Frequency spectrum of output 6GHzpulses

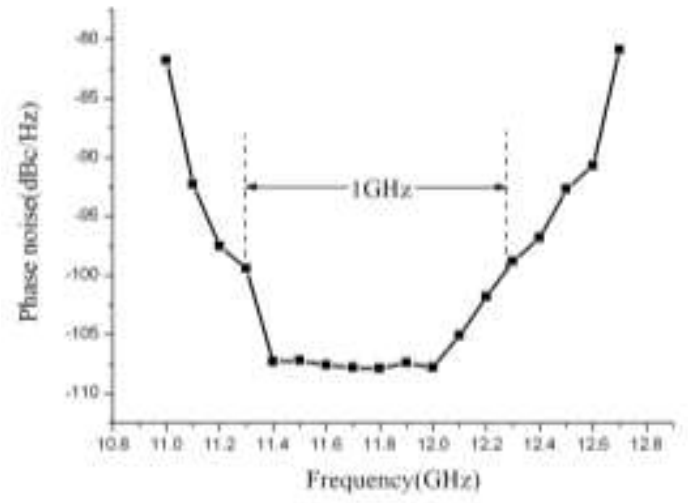

Figure 6. The phase noise of output pulse versus the repetition rate of input 

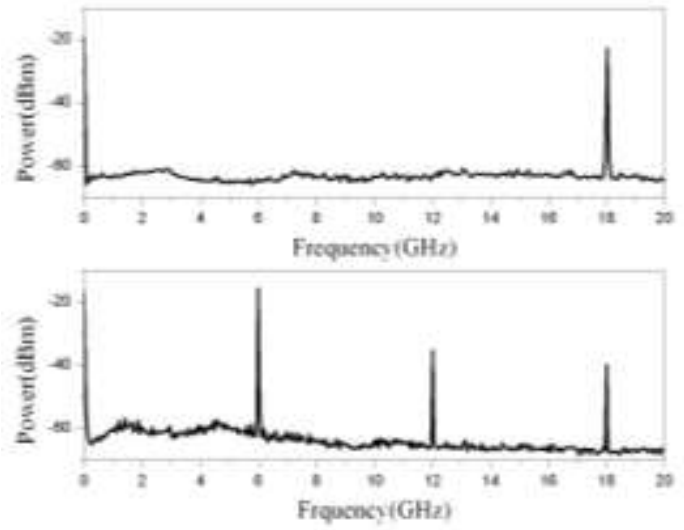

Figure 7. (a) Frequency spectrum of input $18.0 \mathrm{GHz}$ pulses

(b) Frequency spectrum of output $6.0 \mathrm{GHzpulses}$

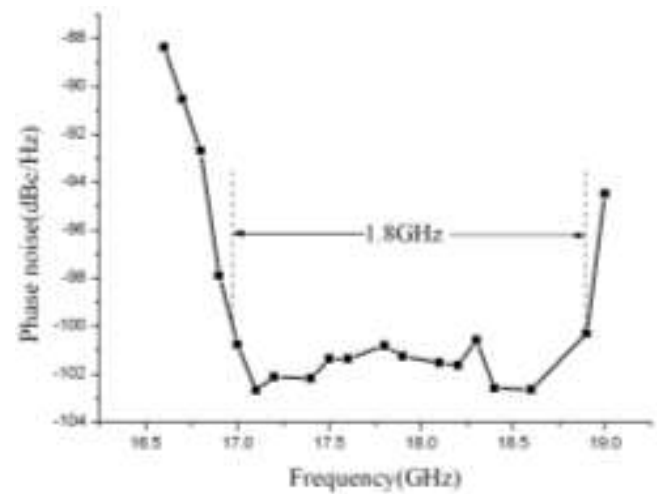

Figure 8. The phase noise of output pulse versus the repetition rate of input optical pulses

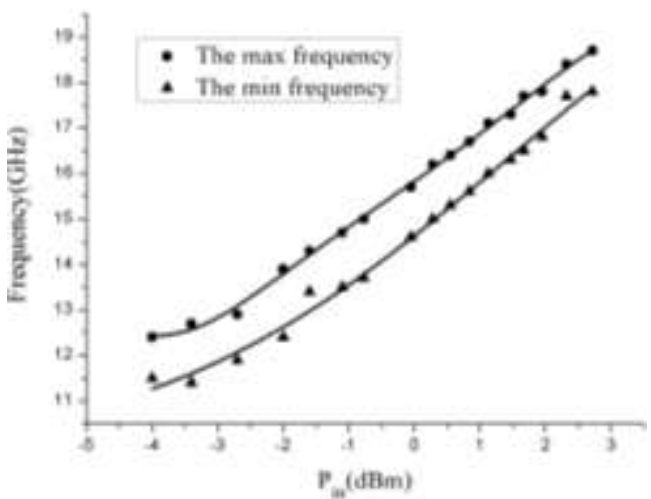

Figure 9. Repetition rate of the input pulse achieved second frequency clock division versus the average power 